\title{
Psychosocial characteristics and needs of mothers with psychotic disorders
}

\author{
LOUISE M. HOWARD, R. KUMAR and GRAHAM THORNICROFT
}

\begin{abstract}
Background It is not known whether mothers with psychotic disorders are clinically and socially distinct from women with psychoses who have not had children.
\end{abstract}

Aims To determine the proportion of mothers in an epidemiologically

representative population of women with psychotic disorders, to examine the factors associated with having children, and to examine the factors associated with having children 'looked after' by social services.

Method Descriptive analysis and two case-control studies.

Results Sixty-three per cent of women with psychotic disorders were mothers. There were no clinical differences between women with or without children, but mothers were more likely to be older and live in unsupported accommodation. Having had a 'looked after' child was associated with Mental Health Act detention, younger age, a forensic history and being Black African.

\section{Conclusion Many women with} psychoses are mothers. Mothers with psychoses are as disabled and have as many needs as women with psychoses without children.

Declaration of interest L. M. H. was funded by the Wellcome Trust as part of a WellcomeTrust ResearchTraining Fellowship in health services research.
Recent reports suggest that significant proportions of women with psychotic disorders have had children and are involved in child care (Test \& Berlin, 1981; White et al, 1995; Gopfert et al, 1996; McGrath et al, 1999). However, research to date has investigated female patients in contact with services, who may not be representative of all women with psychoses who have children. There has been little research into the characteristics and needs of these mothers, even though bringing up a family may bring with it additional risk of relapse, and specific problems and needs that are not addressed by services. Alternatively, mothers may have less severe disorders and higher levels of functioning, and be able to cope with having a family. The role of social support is also not clear, particularly in relation to predicting whether the children of women with mental illness should be 'looked after' by the care system.

We therefore aimed to:

(a) describe the socio-demographic and clinical characteristics of mothers from an epidemiologically representative sample of women with psychosis in south London;

(b) determine the factors associated with having a child in women with a psychotic disorder;

(c) determine the factors associated with having 'looked after' children in mothers with psychotic disorders.

Our hypotheses were, first, that in an epidemiologically representative population of women with chronic psychotic disorders, women with children are more likely to have better psychosocial functioning and have fewer needs than women with no children, and second, that ethnicity, young age, a diagnosis of schizophrenia and being detained under the Mental Health Act 1983 are associated with a history of having had a child placed in care.

\section{METHODS}

The PRiSM Psychosis Study dataset

The PRiSM Psychosis Study was a nonrandomised, controlled trial of two types of sectorised community mental health services in south London and case identification was designed to establish epidemiologically representative cases. The study design has been described extensively elsewhere (Thornicroft et al, 1998a). Cases were found by contacting a wide range of hospital and community services, including social services, primary care, churches, hostels for the homeless and prisons. Available records were screened using the Operational Criteria Check-List (OPCRIT, version 3.2; McGuffin et al, 1991), a structured standardised procedure to generate operationalised research diagnoses. Patients were included in the study if their notes generated an OPCRIT diagnosis of a functional psychotic disorder, that is, schizophrenia (ICD-10 codes F20.0, F20.1, F20.3; World Health Organization, 1992), delusional disorders (F22.0), affective psychoses (F25.0, F25.1, F30.2, F31.2, F31.7, F32.3, F33.3), and non-organic psychoses of unknown aetiology (F29). The Schedules for Clinical Assessment in Neuropsychiatry (SCAN; Wing et al, 1990), a detailed structured standardised clinical interview that generates life-time clinical diagnoses, was administered at Time 1 (1992-1993) to supplement the OPCRIT data in establishing patient diagnosis; other interviews were administered at Time 1 and Time 2 (2 years later) as indicated below.

\section{Instruments}

The study included the following instruments.

\section{Socio-demographic and clinical data}

These data, obtained at the time of the case identification, included number of children, any history of having had a child looked after by the care system, age, age at first illness, gender, ethnicity, social class and family history of mental disorder.

\section{Schedules for Clinical Assessment in Neuropsychiatry (SCAN) (Wing et al, 1990) At Time 1 only. \\ Social Network Scale (SNS) (Dunn et al, 1990)}

This interview measure details total network size and network subgroups (e.g. 
friends, relatives, non-friends (i.e. acquaintances, health staff who are not friends etc.)), and the intensity of interactions (active, passive and intermediate), elicited from the patient during the previous month. Active interactions involve continued verbal interchange, a degree of reciprocity and the presence of the respondent's motivation to be involved. Passive interactions represent a minimal level of interaction - typically the respondent sits alongside someone else without talking or the interchange is limited to a greeting and reply only. Intermediate interactions involve the exchange of articles or the performance of a practical task with/for someone else in the absence of a significant verbal interchange.

\section{Global Assessment of Functioning (GAF) (Endicott et al, 1976)}

This staff interview measures general levels of functioning in the previous month on a 1-90 scale. Total scores above 60 indicate the patient is generally functioning well.

\section{Camberwell Assessment of Need (CAN) (Phelan et al, 1995)}

A scale comprising 22 domains of need which are detailed using separate user and staff assessments.

\section{Client Service Receipt Inventory (CSRI) (Beecham \& Knapp, 1992)}

This measures service utilisation from direct service user interviews.

\section{Lancashire Quality of Life Profile (LQOLP) (Oliver, 1991)}

An interviewer-administered questionnaire with objective and subjective ratings of quality of life in a number of different domains.

Information on substance misuse was generated by collapsing the individual variables from OPCRIT (life-time diagnosis of alcohol or drug dependence, alcohol or drug misuse with psychopathology, alcohol or drug misuse in the index year) into a single variable which was defined as any record of substance misuse on any of these measures.

The data obtained at Time 1 from the schedules above are used for most of the interview data analysis. However, as the CAN was administered to staff and users in both sectors only at Time 2, CAN data for Time 2 are analysed.

\section{Study design}

An initial descriptive analysis was carried out to describe the characteristics and needs of women with children compared with women without children from the case identification and interview data. Two nested case-control studies then examined the factors associated with (a) having children and (b), in those women with children, a history of having had a child looked after by the care system.

\section{Statistical analysis}

Data analysis was carried out using Stata Version 6 (Statacorp, 1999); 95\% confidence intervals $(95 \%$ CIs) are quoted; $P$ values are all two-tailed. A descriptive analysis investigated the distribution of the variables under investigation. Baseline characteristics in women with children and women with no children, and then women with a history of children in care and women with no such history, were compared using $t$-tests for normally distributed quantitative variables, non-parametric tests for quantitative variables that were not normally distributed and $\chi^{2}$ tests for proportions. Logistic regression models were used for multivariate analyses of binary variables and likelihood ratio tests were used to test whether a variable should be included in the model.

\section{RESULTS}

\section{Sample characteristics}

Two hundred and ninety-seven women were identified in the initial case-finding exercise, of whom 261 fulfilled the diagnostic criteria for a psychotic disorder. Case identification data were available for 246 women. The following analyses refer to these women. The median age was 43 years (range 16-89). Two hundred and four $(83 \%)$ women had an OPCRIT diagnosis of schizophrenia, $32(13 \%)$ affective disorder and $10(4 \%)$ other diagnoses. Ninety-five $(39 \%)$ had a clinical diagnosis (in case notes) of schizophrenia, 76 (31\%) had an affective disorder and $74(30 \%)$ had other diagnoses recorded. One hundred and fiftyfive $(63 \%)$ women had had children; 50 women had one child, 45 had two, 32 had three, 13 had four and 15 had had more than 4 children, including one woman who had had eight children. The number of children did not change between the case identification stage and the Time 1 interviews. Data collected by interview at Time 1 confirmed the accuracy of data on the number of children collected from records at the case identification. Two women had one more child each by the time of the Time 2 interviews; none of the women about whom data were collected at Time 2 changed status from being a woman without children to being a mother between Time 1 and 2.

The response rate for interviews and assessments at Time 1 for the 158 women randomly selected for interview varied from $55 \%$ (for the SNS) to $61 \%$ (for the CSRI). There were no significant differences between non-responders and responders to the SNS $(P=0.56$ for clinical diagnosis and $P=0.22$ for ICD diagnosis), detention under a section of the Mental Health Act $(P=0.91)$, GAF score at case identification $(P=0.12)$, living in supported accommodation $(P=0.66)$, employment at case identification $(P=0.16)$, a history of being married $(P=0.19)$, or having had a child $(P=0.15)$.

\section{Social networks}

Social network data were collected on 87 women at Time 1 . Women with children were more likely than women without children to have more contacts with relatives, and less likely to have contacts with acquaintances ('non-friends') and 'intermediate' type contacts (see Table 1).

\section{Needs}

Needs were assessed by the CAN at Time 2 in 75 women, of whom 46 had children. Of the women with children, $22 \%$ (10 of 46 ) rated themselves as having problems with child care. Other significant areas of need (i.e. domains rated as a problem by women with children in more than $20 \%$ of cases) included accommodation (31\%), food (26\%), daytime activities (39\%), physical problems $(37 \%)$, psychotic symptoms $(65 \%)$, information $(29 \%)$, psychological distress $(41 \%)$, company $(37 \%)$, intimate relationships $(29 \%)$, transport $(70 \%)$ and benefits $(32 \%)$

Staff perceptions of needs were similar to those of the women themselves - there was no significant difference in the total number of met needs rated by the users and by staff (paired $t$-test $n=72, P=0.42$ ), although users rated themselves as having more unmet needs $($ mean $=1.67$, s.d. $=1.81)$ 
Table I The social networks of women with and without children

\begin{tabular}{|c|c|c|c|c|c|c|c|}
\hline \multirow[t]{2}{*}{ Type of contact } & \multicolumn{3}{|c|}{$\begin{array}{l}\text { Women without } \\
\text { children }(n=35)\end{array}$} & \multicolumn{3}{|c|}{$\begin{array}{c}\text { Women with } \\
\text { children }(n=52)\end{array}$} & \multirow{2}{*}{$\begin{array}{c}P \text { value } \\
\text { (Mann-Whitney } \\
U \text {-test) }\end{array}$} \\
\hline & Median & (Range) & IQR & Median & (Range) & IQR & \\
\hline Total network size & II & $(4-38)$ & 5,29 & 12 & $(2-33)$ & 3,28 & 0.89 \\
\hline Relatives & 3 & $(0-15)$ & 0,8 & 4 & $(0-24)$ & I,II & 0.04 \\
\hline Friends & 3 & $(0-14)$ & 0,7 & 3 & $(0-20)$ & 0,13 & 0.84 \\
\hline Confidants & 4 & $(0-2 I)$ & 0,9 & 5 & $(0-26)$ & 0,13 & 0.24 \\
\hline Non-friends & 3 & $(0-27)$ & 0,11 & 2 & $(0-16)$ & 0,11 & 0.02 \\
\hline Active interactions & 8 & $(0-35)$ & 3,23 & 8.5 & $(0-3 I)$ & 2,25 & 0.57 \\
\hline Intermediate & I & $(0-7)$ & 0,4 & 0 & $(0-7)$ & 0,4 & 0.01 \\
\hline Passive & 0 & $(0-I I)$ & 0,4 & 0 & $(0-7)$ & 0,3 & 0.64 \\
\hline
\end{tabular}

$\mathrm{IQR}$, interquartile range.

than did staff (mean 1.21, s.d. $=1.85$ ) $(P=0.02)$. Differences in problem areas rated by staff and users $(n=72)$ did occur in the domains of daytime activities $(36 \%$ user ratings compared with $50 \%$ staff ratings), information ( $34 \%$ user ratings, $9 \%$ staff ratings) and benefits $(30 \%$ user ratings, $11 \%$ staff ratings).

Needs (excluding need for child care) were compared for women with and without children, as rated by the women themselves. There was strong evidence that women with children were more likely than women without children to rate themselves as having a problem with intimate relationships (12 $(29 \%)$ women with children had a problem compared with one $(4 \%)$ woman without children; Fisher's exact test $P=0.01$ ). All other domains measured by the CAN did not differ significantly between the two groups. By comparison, ratings by staff of needs in women with and without children ( $n=106$ women, of whom 64 were mothers) showed no differences in any domains.

The user ratings of needs of women with a history of a looked after child $(n=7)$ were not significantly different to those of other mothers $(n=53)$, except that mothers with a history of having a looked after child were more likely to have a problem with child care (Fisher's exact test $P=0.01$ ) and basic education (Fisher's exact test $P=0.03$ ). Staff ratings of needs in these two groups were similar; ratings of the two groups of mothers were not significantly different except that women with a history of a looked after child were more likely to have a problem in the domains of sexual expression (Fisher's exact test $P=0.02$ ) and child care (Fisher's exact test $P=0.008$ ).
There was no evidence of differences in women with and without children in the amount of benefits received, using information from the CSRI at Time 1 $(P=0.24)$. There was some weak evidence of a trend for women with children to have a higher income (median $£ 101.21$; interquartile range $£ 74.89-£ 140.51 ; n=56)$ than women without children (median $£ 82.30$; interquartile range $£ 57.24-£ 131.32 ; n=40$ ) $(P=0.08)$ as measured by the CSRI at Time 1. Analysis of relevant items in the LQOLP inventory showed no difference between these two groups in whether the patient received state benefits $(81-82 \%$ of women in both groups received state benefits; $P=0.96)$ and in the amount of extra money patients felt they needed (Mann-Whitney $U$-test; $P=0.59$ ).

\section{Variables associated with having children}

Having had a child was significantly associated with older age $(P=0.001)$, older age of onset $(P=0.001)$, ever being married $(P<0.001)$, having no qualifications $(P=0.02)$, and also weakly associated with living in unsupported accommodation at the time of case identification $(P=0.09)$. Having a child was not statistically significantly associated with duration of illness $(P=0.31)$, ICD (or clinical) diagnosis $(P=0.64)$, GAF score at case identification $(P=0.98)$, ethnicity $(P=0.45)$, a family history of psychiatric disorder $(P=0.52)$, number of admissions per year $(P=0.24)$, substance misuse $(P=0.59)$, sector $(P=0.96)$, detention under the Mental Health Act $(P=0.26)$, unemployment $(P=0.32)$, history of convictions $(P=0.60)$ or a history of having been in prison $(P=0.90)$. Age and living in unsupported accommodation were the only significant predictors of having a child when the variables above were added stepwise in logistic regression models (see Table 2 for crude and adjusted odds ratios). There was no evidence of an interaction between age (when divided into 10-year age bands) and accommodation (likelihood ratio test $P=0.11$ ).

\section{Variables associated with having a history of a looked after child}

Using data only from patients who had had children, 16 of 155 women $(10 \%)$ had a history of having had a looked after child. Univariate variables significantly associated with having had a child in care were: being Black African $(P<0.001)$ (see Table 3$)$, younger age $(P<0.001)$, younger age of onset $(P=0.01)$, having been charged with any criminal offence $(P<0.001)$, ever having been in prison $(P<0.001)$, ever having been detained under the Mental Health Act $(P=0.004)$, a higher number of admissions per year $(P=0.01)$, duration of illness $(P=0.007)$, living alone $(P=0.006)$, being single $(P=0.002)$, and being a first-generation immigrant $(P=0.01)$. Variables that were not associated with ever having had looked after children included the number of children $(P=0.99)$, substance misuse $(P=0.87)$, diagnosis $(P=0.62)$, having been assessed as at risk of suicide $(P=0.6)$, marital status $(P=0.36)$, a family history of mental illness $(P=0.80)$, education status $(P=0.94)$ and sector $(P=0.63)$.

Logistic regression models were used to investigate possible predictors of having had children in care. All the Black Caribbean and Black African women who had had children in care had also been detained under the Mental Health Act, and detention under the Act therefore could not be included in the logistic regression model. However, using the Mantel-Haenszel test there was still some evidence of an effect of ethnicity on whether children had been taken into care $(P=0.06)$. Black African women appeared to be significantly more at risk of having their children taken into care $-38 \%$ (5/13) of Black African women with children had had children placed in care compared with $3 \%(2 / 73)$ of White women with children. There was also a trend for Black Caribbean women to be 
Table 2 Variables associated with having children $(n=224)$

\begin{tabular}{|c|c|c|c|c|c|}
\hline & No children & I or more children & Crude odds ratio & Adjusted odds ratio & $95 \% \mathrm{Cl}$ \\
\hline Age (mean), years & 40.1 & 48.1 & 1.03 & 1.03 & $1.02-1.05$ \\
\hline \multicolumn{6}{|l|}{ Accommodation } \\
\hline Unsupported & $69(83 \%)$ & $128(91 \%)$ & 0.50 & 0.33 & $0.13-0.80$ \\
\hline Supported & $14(17 \%)$ & 13 (9\%) & & & \\
\hline
\end{tabular}

Table 3 Ethnicity and looked after children

\begin{tabular}{|c|c|c|c|c|c|}
\hline Children ever in care? & White & Black Caribbean & Black African & Other & Total \\
\hline No & $7 \mathrm{ll}$ (97\%) & 35 (81\%) & 8 (62\%) & 3 (75\%) & 117 (88\%) \\
\hline Yes & $2 \quad(3 \%)$ & 8 (19\%) & 5 (38\%) & I (25\%) & $16(12 \%)$ \\
\hline Total & 73 (100\%) & 43 (100\%) & $13(100 \%)$ & $4(100 \%)$ & 133 (100\%) \\
\hline
\end{tabular}

Fisher's exact test $P<0.001$.

Table 4 Final logistic regression model of variables associated with having had a child in care (in women) $(n=|3|)$

\begin{tabular}{lcccl}
\hline Variable & Crude odds ratio & Adjusted odds ratio & $95 \% \mathrm{Cl}$ & $P$ value \\
\hline Age & 0.90 & 0.94 & $0.89-0.96$ & 0.04 \\
History of convictions & 6.67 & 5.12 & $1.53-17.14$ & 0.008 \\
Ethnicity & & & & \\
White (baseline) $(n=7 \mathrm{l})$ & & 1.00 & & 0.15 \\
Black Caribbean $(n=43)$ & 8.11 & 3.49 & $0.65-18.76$ & 0.15 \\
Black African $(n=13)$ & 22.19 & 13.66 & $2.25-83.37$ & 0.005 \\
Other $(n=4)$ & 11.83 & 4.01 & $0.29-56.02$ & 0.30 \\
\hline
\end{tabular}

more at risk of having their children taken into care (8/34 Black Caribbean women had had a child in care).

Likelihood ratio tests were used to decide whether the variables associated with children in care should be included in the model. Age, ethnicity and criminal charges were the only variables associated with having had children in care for women with psychotic disorders in the final regression model (Table 4). However, immigration status was a confounder for the relationship between ethnicity and child care; when immigration status was included in the model there was no longer a significant relationship between ethnicity and having had a child in care - the odds ratio for Black Africans was 3.73 (95\% CI 0.3144.4; $P=0.30$ ) when immigration status was added to the model (likelihood ratio test $\left.\chi^{2}(1)=1.75 ; P=0.19\right)$. First-generation immigrants were, therefore, more at risk of having had their children in care than others and immigration status could replace ethnicity in the model (odds ratio for immigration status $5.74,95 \%$ CI 1.26-26.1; $P=0.02$ ).

\section{DISCUSSION}

\section{Main findings}

Many women with psychotic disorders have children and are involved in child care. Sixty-three per cent of this epidemiologically representative sample of women with psychotic disorders had had children, with most women having had at least two children. A similar proportion (59\%) of mothers was found in a recent Australian study of women in contact with community mental health services (McGrath et al, 1999).

\section{Characteristics and needs of mothers with psychoses}

Women with children were not different clinically from women without children.
Mothers appear to be as disabled, have similar diagnoses, have similarly impoverished social networks and severe illnesses as women without children. Differences in psychopathology that may be associated with child-bearing (e.g. women with negative symptoms may be less likely to have children) should be examined in future studies.

There were also no differences in ethnicity or forensic history, or in needs as measured by the CAN. The data suggest that most of these women live in difficult circumstances with low incomes and few confidants and friends. In addition many women have problems with accommodation, transport, relationships, medical and psychological symptoms and, for women with children, problems with child care. While the PRiSM study excluded patients with only acute and transient episodes of psychosis (F23 in ICD-10) who may have better outcomes, the women in this study are typical of patients in a community mental health team's catchment area.

Mothers had more contact with relatives (which may be for help with child care) and acquaintances (possibly contacts with professionals concerned with children, such as health visitors, social workers) than other women. After adjusting for confounding factors, the only variables associated with having had a child were older age and living in unsupported accommodation.

\section{Factors associated with having a looked after child}

Ten per cent of the women with children had a history of having had a child in the care of social services (i.e. a looked after child). Such a history was more likely in women who had been detained under the Mental Health Act, had a history of convictions, were younger and/or Black African. The ethnic difference could be alternatively explained by immigration status.

Younger age, ethnicity and socioeconomic status are associated with having a looked after child in the general population (Bebbington \& Miles, 1989). The data here are not detailed enough to elicit whether periods in the care system occurred mainly when women were detained in hospital or whether being looked after was a reflection more of chronic parenting difficulties. However, making satisfactory arrangements for child care could be a particular problem when women relapse, 
which may explain why detention under the Mental Health Act is strongly associated with looked after children. Child care responsibilities may make it difficult for women to agree to psychiatric admission voluntarily, despite the impact of maternal illness on the child (Howard, 2000).

Black African women or those women who were first-generation immigrants were more likely to have a history of a child being taken into care. The numbers in these analyses were very small and these results should therefore be interpreted with caution. However, the risk of reception into care for Black African women's children in this study is strikingly high compared with other groups. Immigration status also explained these findings so this may explain some of the ethnic discrepancy. Detention under the Mental Health Act did not fully explain the increased risk among Black Africans of having had children in care, although this was a major explanatory factor. Marital status and living circumstances did not explain these differences, but the data are not detailed enough to identify whether the Black African women are more likely to be single and relatively unsupported at the time of their children being in care.

It is plausible that Black African women who are new immigrants with less social support available to them may ask for their children to be cared for by social services while they find work and appropriate accommodation. However, one study of rates of self-referral to social services did not find differences when comparing White and Black families (Barn 1993). Nevertheless, there is a disproportionate representation of Black (Black African, Black Caribbean and mixed parentage) children in the care system in the general population (Bebbington \& Miles, 1989; Barn, 1993). Previous research has found that Black mothers are more likely to be referred for reasons of mental health by the police and the health service than are White mothers (Barn, 1993). It is therefore likely that future studies may confirm our preliminary findings that Black families with a parent with psychosis are more likely to have children placed in the care system.

As almost all patients were on benefits the socio-economic measures used in this study cannot be used to examine the effect of poverty, but other measures of socioeconomic deprivation may also be important. Alternatively, social services may perceive appropriate parental behaviours differently from the parents' cultural perspective, and language and communication difficulties may increase the gap between social services and the parent, resulting in children being looked after by the care system. Possible interactions between mental illness, ethnicity and socio-economic deprivation and their impact on parenting and the need for children to be looked after by the care system should be investigated in future research.

\section{Methodological limitations}

The epidemiologically representative nature of this sample of individuals with psychotic disorders should have avoided some of the sampling biases of previous studies in this area (Walker \& Lewine, 1993). However, as not all patients were interviewed data collected at case identification were partly dependent on the quality of case notes and staff information rather than information from the patient. The relatively low response rate to interviews is partly due to the inclusion of all prevalent cases (some of whom actively avoid psychiatric services) and the high rates of residential mobility in this population (Thornicroft et al, 1998b). There was no evidence of clinically relevant differences between responders and non-responders, but the similarity between mothers and other women, and comparisons between mothers with looked after children and other mothers, may be due to a lack of statistical power to detect differences.

Differences in functional status between women with and without children were not apparent when examining data from standard instruments such as the global assessment of functioning, or proxy variables for severity of illness such as the number of psychiatric admissions. This may be because these instruments do not measure the more relevant differences in functioning that lead to living with a partner and having children, and future research could investigate which areas of functioning are important for good-enough parenting.

Substance misuse may be underestimated in this study as it was identified through the OPCRIT, which relies on case note information only. At the time of the original study researchers were less aware of the problems and prevalence of substance misuse in patients with psychosis and instruments specifically designed to elicit substance misuse information were not included. However, previous studies (e.g. Menezes et al, 1996) using more detailed sources of information have found rates of substance misuse similar to those found here.

While possible confounders have been included in multivariate models some residual confounding is likely as detailed information on some aspects of patients' problems and needs was not elicited in this study. The age of the patients' children is likely to have an impact on women's needs but detailed information on this was not available. Ratings of ethnicity often fail to reveal the heterogeneity of the groups used in research (Senior \& Bhopal, 1994), but the patients themselves generally agreed with the classification used in this study (Davies et al, 1996). However, there are very small numbers of patients in some of the ethnic groups and these women may not be representative of female patients of these ethnic groups. It is also possible that as Black patients were more likely to be detained under the Mental Health Act child care concerns were more likely to be discussed. However, this does not explain why Black Africans, rather than Black Caribbeans, should be disproportionately found to have had children in care.

The PRiSM Psychosis Study was not designed to investigate differences in women with and without children. Ideally these issues should be examined using a study designed to investigate them, with sufficient power aimed for in the sample size calculation, using predefined measures of outcome to avoid problems of multiple post hoc testing.

\section{Implications}

This study of an epidemiologically representative sample of women with psychosis found that nearly two-thirds of them have had children. These women are just as disabled and have as many (if not more) needs as women with psychosis without children. Support appears to be obtained at least partly from increased contacts with relatives. These findings have implications for these women's mental health, their children's health and for the planning of mental health service delivery. The relationship between ethnicity, social deprivation and needs in these vulnerable women requires more detailed investigation in prospective studies of epidemiologically representative patients with mental illness. 


\section{ACKNOWLEDGEMENTS}

We would like to acknowledge the invaluable contributions that the following colleagues have made towards the PRiSM Psychosis Study: Thomas Becker Sara Bixby, Liz Brooks, Paul Clarkson, Sara Davies, Ruth Fermo, Julie Grove, Hilary Guite, Andrew Higginbotham, Frank Kelly, Julia Kleckham, David Nathniel-Jones, Linda Loftus, Wendy Ojurongbe, Dominic O'Ryan, Sue Parkman, Laura Ponti-Sgargi, Mike Slade, Ruth Taylor, David Turner, Rozalia Wojcik and all the users, carers and staff of the Nunhead and Norwood sectors.

\section{REFERENCES}

Barn, R. (1993) Black Children in the Public Care System. London: British Agency for Adoption and Fostering, Batsford.

Bebbington, A. C. \& Miles, J. B. (1989) The background of children who enter local authority care. British Journal of Social Work, 19, 349-368.

Beecham, J. \& Knapp, M. (1992) Costing psychiatric interventions. In Measuring Mental Health Needs (eds G. Thornicroft, C. R. Brewin \& J.Wing), pp. 163-183. London: Gaskell.

Davies, S., Thornicroft, G., Leese, M., et al (1996) Ethnic differences in risk of compulsory psychiatric admission among representative cases of psychosis in London. British Medical Journal, 312, 533-537.

Dunn, M., O'Driscoll, C., Dayson, D., et al (1990) The TAPS project. 4. An observational study of the social life of long-stay patients. British Journal of Psychiatry, 157, 842-848.

Endicott, J., Spitzer, R. L., Fleiss, J. L., et al (1976) The Global Assessment Scale: a procedure for measuring overall severity of psychiatric disturbance. Archives of General Psychiatry, 33, 766-77I.

Gopfert, M., Webster, J. \& Seeman, M. V. (1996) Parental Psychiatric Disorder. Cambridge: Cambridge University Press.

Hatfield, B., Webster, J. \& Mohamad, H. (1997) Psychiatric emergencies: assessing parents of dependent children. Psychiatric Bulletin, 21, 19-22.

Howard, L. M. (2000) Psychotic disorders and parenting - the relevance of patients' children for general psychiatry services. Psychiatric Bulletin, 24, 324-326.

McGrath, J. J., Hearle, J., Jenner, L., et al (1999) The fertility and fecundity of patients with psychoses. Acto Psychiatrica Scandinavica, 99, 44I-446.

McGuffin, P., Farmer, A. \& Harvey, I. (1991) A polydiagnostic application of operational criteria in studies in psychotic illness: development of reliability of the OPCRIT system. Archives of General Psychiatry, 48 764-770.

Menezes, P. R., Johnson, S., Thornicroft, G., et al (1996) Drug and alcohol problems among individuals with severe mental illness in south London. British Journal of Psychiatry, 168, 612-619.

\section{CLINICAL IMPLICATIONS}

- The majority of women with psychotic disorders have health and social care needs related to pregnancy and child care that may not be recognised by providers of mental health services.

Mothers with psychoses need help in planning alternative care for their children during episodes of relapse.

- A significant proportion of mothers with psychoses will have a child looked after by social services at some point in the child's life.

\section{LIMITATIONS}

Some data were available from case notes only.

- The PRiSM Study was not designed to investigate characteristics associated with mothers with psychoses and this investigation is a post hoc analysis.

- Longitudinal data on factors associated with episodes of children being looked after were not available.

LOUISE M. HOWARD, MRCPsych, Sections of Community Psychiatry (PRiSM) and Perinatal Psychiatry, R. KUMAR, FRCPsych, Section of Perinatal Psychiatry, GRAHAM THORNICROFT, MRCPsych, Section of Community Psychiatry (PRiSM), Institute of Psychiatry, London

Correspondence: Dr L. M. Howard, Sections of Community Psychiatry (PRiSM) and Perinatal Psychiatry, Institute of Psychiatry, De Crespigny Park, London SE5 8AF

(First received I8 May 2000, final revision 13 October 2000, accepted I3 October 2000)

Oliver, J. P. J. (1991) The social care directive: development of a Quality of Life Profile for use in community services for the mentally ill. Social Work and Social Sciences Review, 3, 634-64l.

Phelan, M., Slade, M., Thornicroft, G., et al (1995) The Camberwell Assessment of Need: the validity and reliability of an instrument to assess the needs of people with severe mental illness. British Journal of Psychiatry, 167, 589-595.

Senior, P. A. \& Bhopal, R. (1994) Ethnicity as a variable in epidemiological research. British Medical Journal, 309 327-330.

Statacorp (1999) Stata Statistical Software. College Station, TX: Stata Corporation.

Test, M. A. \& Berlin, S. B. (198I) Issues of specia concern to chronically mentally ill women. Professional Psychology, 12, 136-145.

Thornicroft, G., Strathdee, G., Phelan, M., et a (1998a) Rationale and design. PRiSM Psychosis Study I. British Journal of Psychiatry, 173, 363-370.
_ , Wykes, T., Holloway, F., et al (1998b) From efficacy to effectiveness in community mental health services. British Journal of Psychiatry, 173, 423-427.

Walker, E. F. \& Lewine, R. L. (1993) Sampling biases in studies of gender and schizophrenia. Schizophrenia Bulletin, 19, I-7.

White, C. I., Nicholson, J., Fisher, W. H., et al (1995) Mothers with severe mental illness caring for children. Journal of Nervous and Mental Disease, 183, 398-403.

Wing, J. K. \& Hailey, A. M. (eds) (1972) Evaluating Community Psychiatric Service. The Camberwell Register 1964-1971. London: Oxford University Press.

_ , Babor, T., Brugha, T., et al (1990) SCAN Schedules for Clinical Assessment in Neuropsychiatry Archives of General Psychiatry, 47, 589-593.

World Health Organization (1992) Tenth Revision of the International Classification of Diseases and Related Health Problems. Clinical Descriptions and Diagnostic Guidelines (ICD-10). Geneva: WHO. 